

\title{
Otra escuela es posible: subjetividades políticas y retos en el post-acuerdo
}

Another school institucion is possible: political subjectivities and pos agreement challenges

Outra escola é possível: subjetividades políticas e desafios no pos acordo

Giordano Alvarado Silva Diana Beltrán Bermeo Enrique Blanco García Yesid González Perdomo Javier lbagón Martín 


\section{Giordano Alvarado Silva 1 \\ Diana Beltrán Bermeo 2 \\ Enrique Blanco García 3 \\ Yesid González Perdomo 4 \\ Javier Ibagón Martín 5}

1. Magister en Educación, Tesis Meritoria UPN. Especialista en Gerencia de Proyectos Educativos UDFJC. Licenciado en Lingüística y Literatura UDFJC. Actualmente es docente de planta de la Secretaría de Educación de Bogotá y profesor de medio tiempo de la Escuela de Medios para el desarrollo de la Facultad de Comunicación de Uniminuto; correo electrónico: giordhano@gmail.com

2. Magíster en Filosofía Latinoamericana, Universidad Santo Tomás. Licenciada en Educación Básica con Énfasis en Ciencias Sociales, UDFJ. Educadora popular con el Colectivo Libremente. Actualmente es docente de la Secretaría de Educación de Bogotá y profesora catedrática de la Iberoamericana; correo electrónico: anaidsoc@hotmail.com

3. Magister en Desarrollo Educativo y Social CINDE-UPN, candidato a Magíster en Filosofía Latinoamericana, Universidad Santo Tomás. Educador popular con el colectivo Libremente. Investigador asistente, Universidad Santo Tomás, Facultad de Sociología, grupo Conflictos Sociales, Género y Territorios. Docente de la Secretaría de Educación de Bogotá; correo electrónico: morambo2@gmail.com

4. Magíster en Educación, UPN. Licenciado en Educación Básica con Énfasis en Ciencias Sociales, UDFJ. Docente de la Secretaría de Educación de Bogotá y profesor catedrático e integrante del eje de paz de la UPN, activista sindical; correo electrónico: yesidgonzalezperdomo@gmail.com

5. Magíster en Historia, Pontificia Universidad Javeriana. Realiza sus estudios de doctorado en Ciencias de la Educación en la Universidad de Buenos Aires, Argentina. Docente de la Secretaría de Educación de Bogotá y Coordinador de investigaciones de la Facultad de Educación de la Universidad La Gran Colombia. Integrante del grupo de investigación Educación y Pedagogía (UGC); correo electrónico: goyobraudel@yahoo.com.ar

Fecha de recepción: 18 de abril de 2016 / Fecha de aceptación: 16 de septiembre de 2016

\section{Resumen}

El artículo analiza cinco experiencias pedagógicas desarrolladas en colegios públicos de Bogotá, las cuales reflexionan el conflicto armado, la otredad y los Derechos Humanos, con el fin de transformar las subjetividades políticas de los estudiantes. Realizadas independientemente, vienen consolidándose a través de la Red de Docentes Investigadores del Distrito (Nodo Ciudadanía). Las propuestas comparten el lenguaje artístico como posibilidad de emancipación ontológica y crítica social, necesaria en un país que prepara la finalización del conflicto armado y tiene la responsabilidad frente a los escenarios de confrontación que aún quedan pendientes.

Palabras clave: Escuela, subjetividades políticas, pos acuerdo, otredad, Derechos Humanos, arte.

\section{Summary}

The article analyzes five educational experiences in public schools in Bogotá, which reflect on topics such as the armed conflict, otherness and human rights, with the purpose to transform students' political subjectivities. Developed independently, these have been consolidating through the researchers' network conformed by public school teachers (i.e. Citizenship node). The proposals share the artistic language as a possibility for ontological emancipation and social criticism necessary in a country that is preparing for the end of the armed conflict and the responsibility towards confrontation scenarios still pending to attend.

Keywords: School, political subjectivities, pos agreement, otherness, human rights, art.

\section{Resumo}

Ortigo analisa cinco experiências educacionais em escolas públicas em Bogotá, que refletem o conflito armado, alteridade e os direitos humanos, com o fin de transformar subjetividades políticas de estudantes; Feitas de forma independente, eles são consolidados através da Rede de Professores Pesquisadores do Distrito (Node Cidadania). As propostas compartilham a linguagem artística como possibilidade ontológica de emancipação e crítica social, necessário em um país que se prepara o fim do conflito armado e responsabilidade para com cenários de confrontação ainda pendentes.

Palavras chave: Escola, subjetividades políticas, pos acordo, alteridade, dereitos humanos, arte. 


\section{A manera de introducción}

El presente texto enuncia cinco experiencias que, desde distintos contextos escolares, han venido desarrollando dos tareas fundamentales en la emergencia de la escuela hoy. En primera instancia, están abordando sus prácticas pedagógicas a partir de la experimentación que surge en los procesos de creación y el arte; mientras, al tiempo, piensan en las maneras como se legitiman otros lugares donde también se construye conocimiento. En segundo lugar, se plantean la necesidad de pensar acciones concretas de trabajo colaborativo que permitan visibilizar, fortalecer y conocer dichas experiencias a partir del trabajo en red, en tanto que no solo no están fuera de los posibles escenarios de paz planteados en las negociaciones de La Habana, sino que abordan esta coyuntura histórica desde una perspectiva que media la participación política y la reivindicación de los Derechos Humanos en la escuela.

Desde esta perspectiva, es importante mencionar que las 5 experiencias desarrolladas en este artículo buscan transformar las subjetividades políticas ${ }^{6}$ de sus estudiantes, agenciando mecanismos y estrategias que, desde las expresiones artísticas, construyen lugares posibles para abordar el conflicto armado, la otredad y los Derechos Humanos. En esa medida, visibilizar y conocer estos ejercicios de construcción de ciudadanía (que llevan un trabajo sostenido muy anterior al inicio de los diálogos de paz en Colombia y la promulgación de las leyes que reglamentan su enseñanza: Ley de víctimas y restitución de tierras y la Cátedra para la paz), significa reconocer el papel que ha tenido la escuela y los procesos de enseñanza-aprendizaje a los cuales se ven abocados los maestros y maestras que trabajan en el sector público y privado del país.

A pesar de que las experiencias son muy diversas en términos del abordaje metodológico, acercamiento teórico y producción de contenidos desarrollados por los estudiantes, sí es posible reconocer convergencias en sus intenciones, en última instancia, se trata del motor que ha llevado a escribir este artículo, que parten precisamente de imaginar que las acciones desarrolladas en la escuela, en términos de investigación educativa, tienen una apuesta política que no ha sido visibilizada y que, de manera legalista, se proponen en ejercicios como la Cátedra para la paz y la Ley de víctimas.

Desde esta perspectiva, es la Ley de víctimas la que permite dar un giro a la forma de abordar los temas relacionados con el conflicto armado y la paz, cuando invoca el deber del Estado en la reparación y garantía de no repetición, y propicia condiciones para que la sociedad en su conjunto pueda realizar ejercicios de construcción del pasado. Ahora bien, la Cátedra de la paz, promulgada por Ley 1732 del 1 septiembre del 2014, también interviene en esta tarea, al trazarse como objetivo el fortalecimiento de una cultura de la paz a través de las instituciones educativas.

Teniendo en cuenta lo anterior, es posible relacionar la gestación de las 5 experiencias a partir de realidades específicas identificadas por cada docente en su comunidad educativa. Sin embargo, el espacio académico abierto en la Red Distrital de Docentes Investigadores del Distrito, y más específicamente el Nodo de Ciudadanía, ha permitido establecer un trabajo que busca articularse y que se proyecta para visibilizar dichas prácticas como formas de resistencias a las posturas epistemológicas hegemónicas.

De esta manera, se puede entender la institución escolar desde la pérdida de su protagonismo frente a otros actores en torno al acto educativo (Gvirtz, Abregú y Grinberg, 2007), fundamentalmente en lo que concierne a la enseñanza del conflicto, donde la postura simplista y superficial de los medios masivos de comunicación se impone en la interiorización que desarrollan los estudiantes frente al mismo (Márquez, 2009); esto obliga a la escuela a enfrentar con innovación y compromiso la posibilidad de un país que está a las puertas del fin de una guerra que parecía interminable.

Así pues, podemos inferir que muchas experiencias innovadoras en las aulas de la capital y del país son respuestas anónimas a las actuales exigencias de proyectos que respondan a las negociaciones en La Habana. Sería prudente que tanto las secretarías de educación como el MEN realizarán trabajos para potenciar y sistematizar dichas experiencias, con el objetivo de facilitar el camino que en términos educativos tenemos que recorrer.

\section{Las experiencias}

De esta manera, el trabajo se ha desarrollado en 5 instituciones educativas oficiales de 4 localidades distintas en la ciudad de Bogotá, a continuación se presenta brevemente cada una de ellas:

6 Entendemos por subjetividad política: "aquella manera de estar y de actuar en la organización de su comunidad y de situarse ante ella y ante su organización y administración" (Cristancho, 2012, pp. 9-12). 


\section{Bivokonviviendo ${ }^{7}$}

El proyecto es una iniciativa encaminada hacia la formación en Derechos Humanos y construcción de ciudadanía en el Colegio Kennedy de la Localidad Octava, sus planteamientos se orientan hacia la realización del Foro anual de Derechos Humanos y a la publicación de la revista Ocio para pensar, data del año 2004, cuando se realizó un sondeo entre la comunidad educativa para analizar el conocimiento y la práctica de los Derechos Humanos, con unos resultados no tan satisfactorios.

En primer lugar, no se evidenció una apropiación, promoción y defensa de los Derechos Humanos en los miembros de la comunidad, ni mucho menos un conocimiento de organizaciones e instituciones gubernamentales, o no, que los promuevan y los defiendan; además se presenta un marcado olvido de casos emblemáticos o recurrentes de violación de Derechos Humanos en la comunidad, la ciudad y el país.

Esta problemática impulsó a un grupo de estudiantes, docentes, madres, padres y administrativos, a realizar un primer Foro titulado: "Derechos Humanos y Formación Ciudadana". El comité organizador recogió el trabajo propuesto desde la labor interdisciplinar en las aulas, destacando ponencias en torno a los imaginarios sociales de la comunidad y sus problemáticas, cerrando con un manifiesto de acciones que debía desarrollar la comunidad para mejorar la interiorización y práctica de los Derechos Humanos dentro y fuera de la institución educativa.

Vale la pena mencionar que el proyecto Bivokonviviendo ha realizado el foro de manera ininterrumpida hasta el presente año, con la participación destacada de víctimas, académicos, defensores de Derechos Humanos, funcionarios públicos, integrantes de comunidades educativas de otros colegios y, por supuesto, miembros de la comunidad educativa del colegio Kennedy IED. De la misma manera, cabe resaltar que durante los últimos 3 años las temáticas abordadas por el foro y la revista giraron en torno a la

7 La palabra Bivokonviviendo se construye haciendo alusión al griego Bios (Borisonik y Beresñak, 2012), que nos indica vida activa y defensa de la misma, el rechazo a cualquier agresión a partir de la construcción de elementos y métodos para su cuidado, desde lo individual hasta lo colectivo. La siguiente palabra es "konviviendo", término que manifiesta convivencia y respeto por la diferencia; se desarrolla en nuestra comunidad educativa, que está atravesada por tensas situaciones cotidianas, que dependen en su mayoría de los contextos sociales problémicos de los integrantes de la institución. Se escribe con la letra K por la cimentación de nuestra identidad y memoria, porque nuestro colegio acoge el apellido del asesinado Presidente de los Estados Unidos John F. Kennedy. paz y el conflicto armado, como un aporte más en la construcción de una sociedad incluyente y sin guerra.

Para el año 2010 el proyecto puso en marcha un medio de expresión y difusión, este trabajo llevó al nacimiento de la revista Ocio para pensar, una publicación llena de esperanzas y sueños que recoge las vivencias, experiencias, producción académica y éxitos de un proyecto que se consolida cada año como una manifestación alternativa, de una comunidad preocupada por la solución de las principales problemáticas del país.

\section{¿Qué tienes en la mente? Narrativas contemporáneas, prácticas artísticas y configuración de la subjetividad en jóvenes}

Este trabajo recoge la experiencia artística y audiovisual de un grupo de jóvenes que participa en el proyecto de comunicación y medios escolares del Colegio Nueva Delhi de la localidad cuarta, los cuales han encontrado en las prácticas artísticas nuevas maneras de asumir el sentido y su subjetividad. Más allá de sistematizar una experiencia, el ejercicio está encaminado a redimensionar los procesos de investigación educativa, en tanto que no solo se trata de procesos que se interesan por indagar marcos metodológicos, pedagógicos o conceptuales, sino que, incluyendo eso, también dan un lugar preponderante a la idea de la creación como un escenario donde también se construye el conocimiento.

Así, además de hacer un análisis de cómo las sociedades han configurado el sentido a lo largo de la historia (desde el mito hasta las industrias mediáticas contemporáneas), este trabajo busca conectar otros caminos y sistemas simbólicos que persisten en los procesos de enseñanza-aprendizaje. Bajo este panorama, se emplea la investigación-creación como una herramienta metodológica que permite explorar el interés, de los y las jóvenes, por el impacto de los desarrollos tecnológicos en la vida cotidiana y las maneras en que las prácticas artísticas contribuyen a construir otras formas de entender las relaciones y significados que se tiene de uno mismo y del territorio.

En efecto, esta experiencia plantea la idea de dimensionar de otro modo el papel de lo mediático en el mundo actual, y el papel de las prácticas artísticas en la configuración de los sujetos y la subjetividad. Lenguajes sonoros, plásticos y/o audiovisuales, buscan experimentar otros sistemas de representación, donde los sujetos ya no solamente se limitan a observar las producciones hechas por las industrias culturales, sino que asumen en las prácticas del arte un rol distinto dentro de la producción de nuevas maneras de narrar el mundo en el que viven. 
El proyecto de comunicación “¿Qué tienes en la mente?” es el producto de una investigación -creación que, desde 2011, ha planteado la idea de la producción de contenidos hechos por los estudiantes como un escenario de construcción de sentido. De la misma manera, año tras año se desarrolla el festival de comunicación escolar, donde se dan a conocer los trabajos hechos por los estudiantes en los diferentes lenguajes artísticos, así como la participación en convocatorias distritales con la $\mathrm{SED}^{8}$. Este trabajo asume los procesos de creación como lugares donde se construye conocimiento, porque al igual que la ciencia: "la actividad artística (...) fabrica imágenes, objetos e ideas sobre lo real" (Laiglesia, 2008).

\section{Deconstruyendo alteridades: hacia el reconocimiento histórico de lo(s) otro(s)}

A partir del reconocimiento de la alteridad como base sustantiva de procesos formativos tendientes al fortalecimiento de una cultura de paz en la escuela, desde hace dos años se viene desarrollando

8 Producción de Cortometraje de ficción en Mi Edu en Serie, en alianza con el Canal Capital y la Secretaría de Educación del Distrito. Participación en La NETT (Nuestras Escuelas Transforman Territorios), en alianza con Señal Colombia y la Secretaría de Educación del Distrito; nominada a los premios India Catalina 2016 como Mejor Programa Juvenil. un proyecto de aula con estudiantes de básica secundaria y media del Colegio Clemencia de Caycedo IED, ubicado en la localidad dieciocho, que busca identificar silencios, omisiones y "encubrimientos" (Dussel, 1994) en los discursos oficiales acerca de la historia de Colombia.

En este sentido, la iniciativa "Deconstruyendo alteridades: hacia el reconocimiento histórico de lo otro", se ha centrado en establecer escenarios escolares de discusión que propendan por la identificación de discursos y prácticas hegemónicas desde las cuales, en medio de los diversos procesos históricos de estructuración de una identidad nacional homogénea, se ha "inventado al otro" en Colombia (Castro-Gómez, 1993) negándolo y estereotipándolo.

Dicho ejercicio crítico se considera fundamental en contextos socio-políticos en los que el tema de la paz se ha configurado como un eje transversal para estructurar un horizonte de país, ya que los procesos de reconciliación que se buscan desarrollar en el presente deben estar estructurados desde la revisión y re-significación de un pasado que, discursivamente, ha servido para excluir y marginar lo(s) otro(s).

Metodológicamente hablando la propuesta se ha cimentado en la investigación-acción, sustento que ha facilitado la construcción de ejercicios de reflexión crítica que continuamente se están replanteando por parte del docente y las estudiantes. En la primera fase se ha ubicado como eje central de discusión y análisis

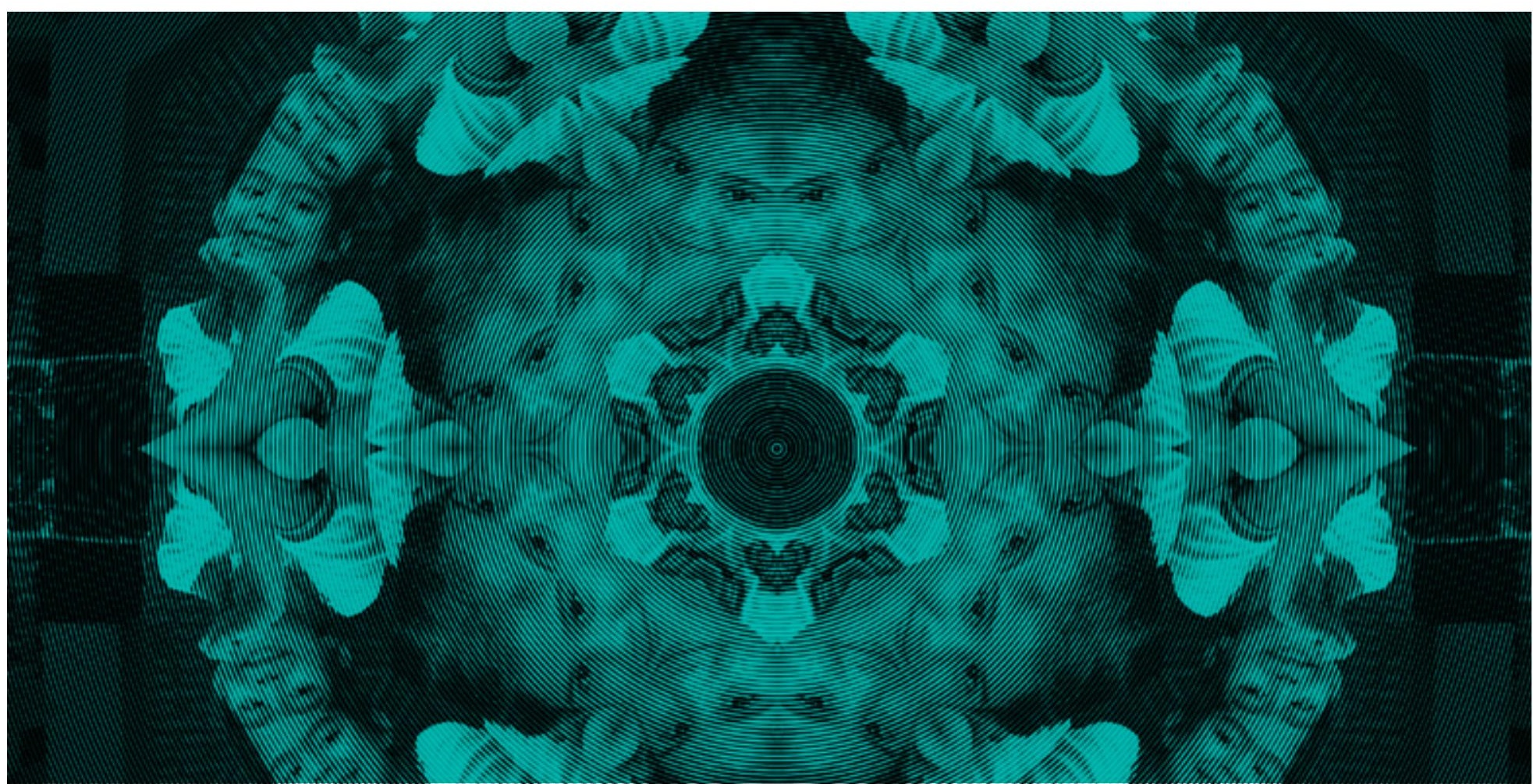


el problema de lo afrocolombiano, puesto que aunque indígenas, campesinos, mujeres y sectores populares hacen parte de los grupos que fueron negados sistemáticamente por la historia oficial, la matriz socio-cultural africana ha sido especialmente estereotipada e invisibilizada, configurándose como un "último otro" (Muteba, 2013) en la construcción narrativa de la historia del país y su posterior enseñanza.

A partir de este escenario de discusión las estudiantes han venido reconociendo críticamente otras voces y agencias, develando el discurso científico y político dominante. Crítica que se ha gestado a través del uso de diferentes lenguajes artísticos, entre los cuales cabe destacar: el teatro (adaptación de cuentos, construcción de guiones), la pintura (muestras itinerantes) y la música (recuperación de la tradición musical).

\section{Observatorio Escolar de Derechos Humanos (OEDHU)}

Este trabajo surge en 2012 en el Colegio Técnico Tomás Rueda Vargas IED, desde el área de ciencias sociales; durante este año se hizo una revisión bibliográfica, un ejercicio de diagnóstico con los estudiantes y varias reuniones entre los maestros autores de la propuesta, para indagar sobre la necesidad y pertinencia de la actividad. En 2013, con las "Iniciativas ciudadanas para la transformación de realidades", INCITAR, se presenta formalmente la propuesta, siendo evaluada y admitida. Durante 2014 y 2015 se dio el proceso de consolidación, realizando talleres, exposiciones, construcción de imágenes fotográficas, cortometrajes y una salida pedagógica al Centro de Memoria, Paz y Reconciliación. Dichas actividades produjeron un archivo que está en proceso de análisis y sistematización.

Esta experiencia se ha construido para atender la dificultad de visibilizar la práctica y el ejercicio real de los Derechos Humanos, evidenciada en las diferentes formas de violencia al interior del escenario escolar. El objetivo general es hacer visible el ejercicio y garantía de los DD.HH en la institución a partir de situaciones cotidianas. De esta forma, se identifican las prácticas sociales presentes en el currículo oculto, así como la formulación de instrumentos, alternativas y soluciones a las problemáticas presentes en el colegio y que podrían ser pensadas en espacios de integración curricular.

En primer lugar se busca poner en diálogo el paradigma de los DD.HH con la cotidianidad escolar, mediante un escenario que tenga como propósito la investigación de la realidad de los estudiantes y las múltiples expresiones de sus problemáticas. Este

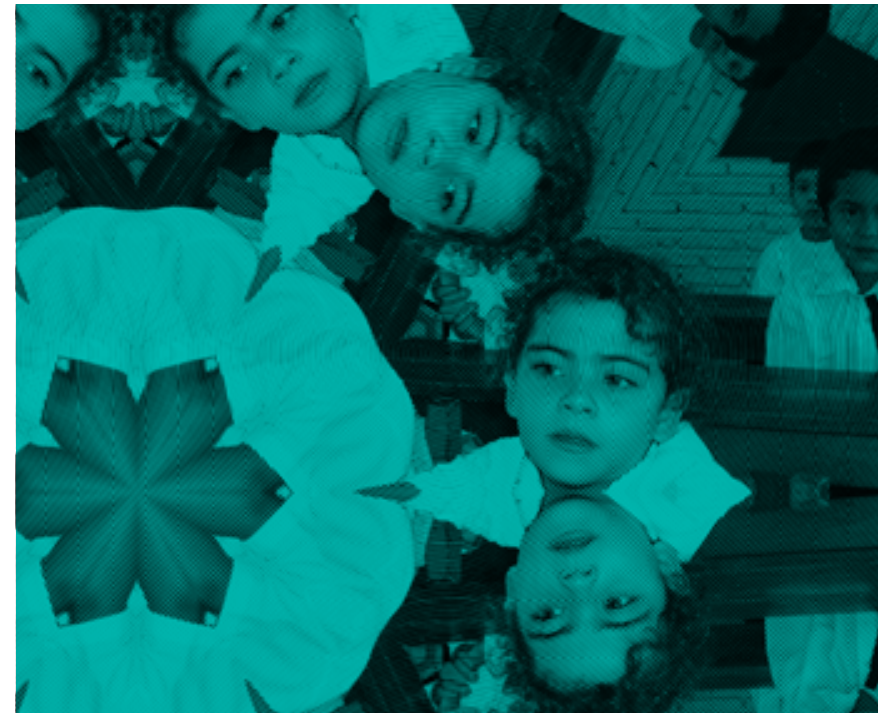

escenario se ha denominado "Observatorio Escolar de Derechos Humanos" O.E.D.H.U., y con él se busca responder la pregunta ¿Promueve el colegio la garantía y el goce pleno de los DD.HH? Los docentes y estudiantes son los encargados de planificar y organizar tiempos, actividades, resultados esperados y análisis de la información recolectada. Desde el observatorio se busca propiciar un diálogo ético, político y crítico que cuestione las relaciones sociales hegemónicas que configuran la subjetividad.

La propuesta filosófica de dialéctica del reconocimiento, la pregunta por el otro como compromiso ético y la pedagogía crítica como praxis política, fueron puestas en diálogo constante mediante una planeación que debe ser revisada y actualizada continuamente. El proyecto fue formulado tomando como discurso artístico y cultural a la fotografía, vista como herramienta de observación social y como posibilidad de producción creativa emancipadora. Si las funciones básicas de un observatorio son la observación y la intervención social, la imagen fotográfica es el dispositivo de captura de dicha realidad; Barthes, Eco, Lefebvre, Giddens, entre otros, alimentan conceptos como cotidianidad, recuerdo y representación, para dar vida al relato como el aura de la fotografía, tal como lo dijera Walter Benjamin. 
Contando y pintando: Una mirada a los Derechos Humanos y el conflicto armado colombiano a través del arte y la literatura. Una estrategia para la construcción de paz y ciudadanía

Esta propuesta pedagógica se viene desarrollando desde 2010 en el colegio Leonardo Posada Pedraza IED, localidad de Bosa, surge en el seno del área de Ciencias Sociales y su objetivo fundamental es fortalecer las capacidades ciudadanas ${ }^{9}$ bajo una apuesta ética orientada en el reconocimiento y acogida del otro; fue pensada con dos fines prácticos específicos:

- El rescate de los espacios públicos de la institución a través de actos comunicativos simbólicos y artísticos.

- La promoción de posturas críticas de los estudiantes frente a la realidad nacional, a partir de la exploración y producción textual y literaria.

La experiencia se ha desarrollado en tres etapas, las dos primeras: "Rompiendo paredes" y "El arte en la mesa" (2010-2014), centraron su atención en la apropiación y transformación de los espacios institucionales, haciendo uso del enfoque pedagógico institucional, en este caso La Enseñanza Para la Comprensión ${ }^{10}$. De acuerdo al enfoque, la fase final del proceso enseñanza-aprendizaje potencia la dimensión comunicativa por medio de proyectos finales de síntesis que dan voz a los estudiantes, quienes hacen creaciones simbólicas y artísticas plasmadas en muros y mesas de la institución, que fueron resultado de la reflexión en torno a los Derechos Humanos y el Conflicto armado colombiano. El ejercicio incrementó el uso de otros lenguajes y herramientas en las etapas de exploración e investigación; la literatura, el cine, la música y la pintura son imprescindibles, nutriendo a las ciencias sociales.

La tercera fase: "Foro Institucional de Derechos Humanos Leonardo Posada Pedraza" (2015), se transforma en una apuesta abierta a otras áreas de conocimiento, específicamente el área

9 Entenderemos por capacidades ciudadanas la definición propuesta por el Plan Integral para Ciudadanía y convivencia PIECC, como: aquellos conocimientos, actitudes, habilidades, motivaciones y prácticas que realizan los sujetos de las comunidades educativas, para reflexionar, hacer, ser, estar, conocerse y reconocer su contexto, para imaginar su transformación y actuar con otros para transformarlo (SED, 2015)

10 Esta apuesta pedagógica se desarrolla en tres etapas: exploración (Reconocimiento inicial de los conceptos a comprender); investigación guiada (Desarrollo de la comprensión de los conceptos) y desarrollo del proyecto final de síntesis (Los estudiantes sintetizan y demuestran la comprensión alcanzada respecto al tópico generativo). de humanidades, para propiciar el diálogo de saberes y potenciar la producción textual y literaria en los estudiantes, así como el diálogo con la comunidad educativa y entre pares de diferentes localidades; es un ejercicio académico que mantiene la voz de los estudiantes como eje central a través de sus múltiples manifestaciones, dando origen al primer número de la revista Mnemosine, contando y pintando por la ciudadanía y la paz, que recoge algunas producciones textuales y literarias de los estudiantes respecto al conflicto armado en Colombia.

\section{De los puntos de encuentro y los retos}

El encuentro suscitado por la convocatoria de la Red de Docentes Investigadores ha permitido el diálogo entre los profesores que lideran las anteriores experiencias, propiciando la reflexión colectiva, la discusión académica y la proyección a un futuro próximo. Las disertaciones respecto a las experiencias pedagógicas abonan a la discusión frente a conceptos y problemas que históricamente han buscado reflexionar sobre la educación, cómo se construyen las ciudadanías y el problema filosófico del conocimiento, ejes centrales del proceso de enseñanza-aprendizaje en una apuesta de educación para el desarrollo humano.

En este sentido, la escuela como institución moderna ha sido el espacio privilegiado en la formación de la subjetividad, expresada en la ciudadanía como discurso y práctica social necesaria para alcanzar las promesas de la modernidad. Sin embargo, este proceso desembocó en la homogeneidad y estandarización del sujeto escolar, invisibilizando otras formas de ser y estar en el mundo, produciendo interrogantes y una crisis innegable al interior de la escuela, expresada en la pérdida de sentido sobre la necesidad de la educación como condición para la emancipación, en la imposibilidad de responder a los retos de la sociedad y en la anulación del pensamiento crítico transformador.

De esta forma, el proyecto liberador de la escuela fue desplazado por la racionalidad de la individualidad, bajo la figura del egoísmo y el éxito cuantitativo-numérico como sinónimo de progreso en los procesos de medición y evaluación escolar, por la producción de riqueza material donde el ser económico se presenta como la única alternativa existencial, y por la competitividad como imperativo ético en un mundo cada vez más excluyente y violento.

En cambio, las propuestas enunciadas pretenden pluralizar el concepto de ciudadanía y evidenciar nuevos espacios en la 
formación de subjetividad, a partir de la creación artística como paradigma trasgresor y posibilitador de otras maneras de entender y ejercer la ciudadanía. Por lo tanto, el arte en todo su potencial humanizador sería un elemento fundamental de la práctica pedagógica, trascendiendo los requerimientos de medición y competitividad con los que se lee y evalúa la praxis educativa en la actualidad.

La ciudadanía desde esta perspectiva estaría compuesta por el agenciamiento e impacto que el estudiante pueda tener en su contexto próximo, reflexionando continuamente sobre sí en relación con otros, en un movimiento dialéctico que, a partir de la creación, pueda construir nuevas experiencias y escenarios de reconocimiento intersubjetivo, en un país que pretende hoy pensar en serio la paz y la reconciliación como horizontes de futuro.

Por consiguiente, es evidente que sin duda alguna existe, en las experiencias presentadas, una apuesta epistemológica que desborda los límites impuestos por la racionalidad occidental. Esta situación nos convoca a la reflexión respecto de la imposibilidad de un conocimiento único y limitado, y pone sobre la mesa la discusión en torno a la alternativa de construcciones epistémicas a partir de los aportes de diferentes disciplinas. La crisis innegable del paradigma epistemológico dominante durante el último siglo, parece abrir la brecha para facilitar la de-construcción del legado moderno, la endiosada razón que, en el seno del positivismo, guió los caminos del conocimiento es una condición revalidada hoy, dado que se constituye como lectura insuficiente de una realidad compleja:
Los diferentes niveles en que se nos presenta la realidad, en todos los campos, pero, de una manera especial, en la realidad de los seres vivos, exigen también diferentes niveles de la lógica a aplicar y, en nuestro caso, una dialógica transdisciplinaria y unos métodos también transdisciplinarios (Martínez, 2011, p. 15).

La posibilidad de construcción de conocimiento, de la mano con procesos creativos, nos coloca ante retos coyunturales que atraviesan la ciencia misma, y nos exige re-pensar el conocimiento y la práctica pedagógica. Los retos giran en torno a las posibilidades que nos abre hoy la escuela (sin desconocer otros escenarios y protagonistas del acto educativo), hacen evidente la necesidad de los docentes por iniciar trabajos de sistematización y socialización de las experiencias significativas en el aula, más aún cuando pretenden aportar en el desescalamiento del conflicto y la posibilidad de una generación de paz.

Por último, vale la pena mencionar la exigencia que tenemos de fortalecer los escenarios de trabajo en red, ya que además posibilitar la reflexión y escritura colectiva de las experiencias, han generado un cronograma de actividades que busca ampliar la divulgación y participación de las comunidades educativas en los distintos escenarios donde se desarrollan las experiencias. 


\section{Referencias}

Barbero, J. M. (2002). La educación desde la comunicación. Eduteka, pp. 1-17.

Borisonik, H., y Beresñak, F. (2012). Bíos y Zoé: una discusión en torno a las prácticas. Astrolabio. Revista internacional de filosofía, pp. 82-90.

Bourriaud, N. (2008). Estética relacional. Buenos Aires: Adriana Hidalgo Editora.

Castro-Gómez, S. (1993). Ciencias sociales, violencia epistémica y el problema de la "invención del otro". En Lander, E. (Comp.). La colonialidad del saber: eurocentrismo y ciencias sociales. Perspectivas latinoamericanas (pp. 145-161). Buenos Aires: CLACSO.

Cristancho A., J. G. (2012). Los conceptos, sujetos y subjetivación política-propedéutica para una reflexión. Bogotá: Universidad Pedagógica Nacional.

Dussel, E. (1994). 1492 el encubrimiento del otro. Hacia el origen "del mito a la modernidad". La paz: UMSA-Plural editores.

Eisner, E. W. (2002). La escuela que necesitamos. Buenos Aires: Amorrortu.

Goodman, N. (1976). Maneras de hacer mundos. Barcelona: Seix Barral.

Gvirtz, S., Abregú, V., y Grinberg, S. (2007). La educación ayer, hoy y mañana- El ABC de la Pedagogía. Buenos Aires: Aique.

Laiglesia, J. F. (2008). Notas para una investigación artística. Pontevedra: Universidad de Vigo, Servicio de publicaciones.

Larrosa, J. (2005). Tecnologias del Yo y educación (notas sobre la construcción y la mediación pedagógica de la experiencia de sí). Buenos Aires: Miño y Dávila Editores.
Márquez, M. (2009). Enseñanza de la historia del conflicto armado en Colombia: Fundamentos para la construcción de propuestas para su enseñanza en el ámbito universitario. Revista latinoamericana de estudios educativos, pp. 205-230.

Martínez Miguelez, M. (2011). El paradigma sistémico, la complejidad y la transdisciplinariedad como bases epistémicas de la investigación cualitativa. Revista electrónica de humanidades. Educación y comunicación social REDHECS, 11, pp. 6-27.

Muteba, J. (2013, septiembre 25). Movimientos sociales negros en América Latina, el giro hacia el multiculturalismo y "nuevas" conceptualizaciones de la diáspora africana. En Conferencia Inaugural Terceras Jornadas de Estudios Afro latinoamericanos del GEALA, Buenos Aires: GEALA (Mimeo).

República de Colombia. (2011). Ley de víctimas y restitución de tierras. Bogotá: Imprenta Nacional.

Rincón, O. (2010). Tácticas y estrategias para contar. Bogotá: Fundación Friedrich Ebert en Colombia.

Secretaría de Educación Distrital. (SED). (2015). Planes Integrales de Educación para la ciudadanía y la convivencia. Obtenido el 21 de Agosto de 2015, desde http://www.redacademica.edu.co/archivos/redacademi$\mathrm{ca} /$ proyectos/pecc/centro_documentacion/caja_de_herramientas/ serie_1_orientaciones/06_planes_integrales_de_educacion_para_la_ ciudadania_y_la_convivencia_piecc.pdf

Thompson, J. (1998). Los media y la modernidad. Una teoría de los medios de comunicación. Barcelona: Paidós Ibérica.

Verón, E. (1993). La Semiosis Social. La Semiosis Social. Fragmentos de una teoría de la discursividad. Barcelona: Gedisa. 